\title{
Relation of Magnet, Current and Structure of Matter
}

\author{
Rajatava Mukhopadhyay \\ Howrah, West Bengal, India
}

\begin{abstract}
Magnet, current and structure of matter are intensely correlated.A simple experiment and deductions based on that experiment demonstrates that.
\end{abstract}

Keywords: magnet

\section{Introduction}

Oesterd's experiment and subsequently Faraday's kicking wire experiment has already chalked out some ideas about the relation magnet and current and their effects on one another. In this paper one will get introduced to the relation between magnet, current and structure of matter alike with the help of a simple experiment. So without any further delay let us move on to the experiment.

\section{Objective of Experiment}

To prove the link between electric charge and magnetic poles and independent existence of isolated magnetic poles.

\section{Apparatus Required}

A solution of common salt in distilled water, with a few drops of $\mathrm{HCl}$ is taken in a vessel. A bar magnet is dropped into it.

Observation: Small bubbles are found accumulating near the 2 poles of the magnet. After sometimes, small bubbles merge together to form a bigger bubble. This bigger bubble eventually escapes from the surface of the magnet. The bubbles are colourless and odourless. Rate of evolution of bubble at the north pole is less than that at the south pole.

Inference: Weber's theory speaks of every minute particle being a magnet, provided the particle being one of a magnet or magnetic substance. Based on this theory, $\mathrm{Fe}^{3+} / \mathrm{Fe}^{2+}$ ions were proved and verified to be attracted by a magnet.

Oesterd's experiment proved the direction of magnetic field in a conductor from its -ve to + ve terminal. So we understand that a temporary north pole is developed at the ve terminal and south pole at the +ve terminal.

Therefore,

+ ve terminal $=$ cluster of cations $=$ isolated South Pole

-ve terminal $=$ cluster of anions $=$ isolated North Pole

So, any molecule/atom will be a complete magnet can be justified.

From the components present in the set-up and appearance of the bubbles, the bubble at South Pole appears to be $\mathrm{O}^{2-} / \mathrm{OH}^{-}$ $/ \mathrm{Cl}^{-}$and that at North Pole is $\mathrm{H}^{+}$. These clusters of ions when become big enough and when the Coulombian force becomes more intense than magnetic force of attraction, they become neutralized and escape from the surface of the magnet.

So from here, another important inference may be drawn:

1) More electropositive any element is, the less is the magnetic force of attraction of its cation and vice-versa.

2) More electronegative any element is, the less is the magnetic force of attraction of its anion and vice-versa.

Therefore, in the light of this experiment, structure of matter (or atom) may also be justified.

Finally, it may be stated that after 2 hours of the beginning of the experiment, the solution level in the vessel is found to decrease by $0.5-1 \mathrm{~mm}$.

\section{Conclusion}

So, this experiment also infers that magnetism and electricity are co-incident, better said they are different aspects of one happening.

\section{References}

[1] Fundamentals of Physical Science (IX-X) by Sisir Ranjan Majumdar

[2] Concepts of Physics 2 by Harish Chandra Verma

\section{Author Profile}

Rajatava Mukhopadhyay is in South Point High School, Kolkata, India 